\title{
Anti-Inflammatory Pre-Treatment to Reduce Mobilization-Induced Liver Inflammation in Mice: Novel Model to Study Liver Injury
}

\author{
T. Hendrikx ${ }^{1,2}$, C. Piersma ${ }^{3}$, D. K. Dhar ${ }^{4,5}$, L. Mpabanzi' ${ }^{2,3}$, J. J. W. Schreurs ${ }^{1,2}$, M. Malagó4, \\ R. Shiri-Sverdlovi,2, S. W. M. Olde Damink ${ }^{2,3,4^{*}}$ \\ ${ }^{1}$ Department of Molecular Genetics, Maastricht University, Maastricht, The Netherlands \\ ${ }^{2}$ Nutrition and Toxicology Research Institute Maastricht, Maastricht University, Maastricht, The Netherlands \\ ${ }^{3}$ Department of Surgery, Maastricht University Medical Centre, Maastricht, The Netherlands \\ ${ }^{4}$ Department of HPB Surgery and Liver Transplantation, Royal Free Hospital, University College London, London, \\ UK \\ ${ }^{5}$ Comparative Medicine Department, Organ Transplantation Center, King Faisal Specialist Hospital \\ and Research Center, Riyadh, Saudi Arabia \\ Email: ${ }^{*}$ steven.oldedamink@maastrichtuniversity.nl
}

Received 28 April 2015; accepted 19 May 2015; published 25 May 2015

Copyright (C) 2015 by authors and Scientific Research Publishing Inc.

This work is licensed under the Creative Commons Attribution International License (CC BY). http://creativecommons.org/licenses/by/4.0/

(c) (i) Open Access

\section{Abstract}

Background: Liver surgery requires mobilization of the liver resulting in injury. Mobilization is the predominant cause of hepatocyte damage during liver surgery and jeopardizes post-operative liver function. Previously, mobilization-induced liver injury was found to be associated with inflammation. So far, anti-inflammatory drugs to potentially prevent liver inflammation following liver mobilization were not tested. In this study, we aimed to establish an in vivo mouse model of mobilization-induced liver injury and to evaluate the effect of anti-inflammatory pre-treatment before liver mobilization on liver inflammation. Methods: To develop a mouse model for mobilization-induced liver injury, C57BL/6 mice $(n=8)$ underwent surgery during which the liver was mobilized by gentle manipulation of the lobes with cotton-wool applicators for 15 minutes. In two control groups, the liver was left alone or was subjected to laparotomy only. An additional group was added that received anti-TNF treatment (Infliximab) 2 days prior to surgery. Liver samples were obtained 2 hours after mobilization and liver inflammation was analyzed by histology and inflammatory gene expression. Results: Gentle liver mobilization resulted in acute liver inflammation as indicated by increased recruitment of inflammatory cells and elevated inflammatory gene expression compared to controls. Infliximab pre-treatment had no effect on the inflammatory response in the liver. Conclusion: Our current model provides an excellent opportunity to study the effects of pre-treatment with anti-inflammatory drugs on mobilization-induced liver inflammation.

\footnotetext{
${ }^{*}$ Corresponding author.
}

How to cite this paper: Hendrikx, T., et al. (2015) Anti-Inflammatory Pre-Treatment to Reduce Mobilization-Induced Liver Inflammation in Mice: Novel Model to Study Liver Injury. Modern Research in Inflammation, 4, 1-7.

http://dx.doi.org/10.4236/mri.2015.41001 
Further studies are needed to investigate whether anti-inflammatory mediators can be used to prevent liver inflammation.

\title{
Keywords
}

\author{
Liver Surgery, Hepatocellular Damage, Inflammation, Translational Model, Post-Resectional Liver \\ Failure
}

\section{Introduction}

During hepatic surgery, it is mandatory to dissect the liver from surrounding ligaments, a procedure known as "liver mobilization". During mobilization it is necessary to maneuver, manipulate and retract the liver to have a better view of the dissection area. However, previously it has been shown that this procedure often results in liver damage due to squeezing of the organ, resulting in post-operative problems in patients undergoing hepatic surgery [1]-[3].

In humans, we have previously demonstrated that liver mobilization is the predominant cause of hepatocyte damage during liver surgery [4]. As shown by infiltration of inflammatory cells and up-regulation of genes involved in acute inflammation, liver mobilization was found to be associated with hepatocellular damage and liver inflammation [4] [5]. So far, the potential protective effect of anti-inflammatory pre-treatment in an in vivo mouse model of mobilization-induced liver injury has not been investigated.

In this study, our aim was to establish a mouse model to study mobilization-induced liver injury in vivo. Additionally we aimed to assess the effect of anti-inflammatory pre-treatment on liver inflammation after mobilization. As tumour necrosis alpha (TNF- $\alpha$ ) is an important pro-inflammatory cytokine released by Kupffer cells [6] and Kupffer cells were shown to play an important role during mobilization-induced liver inflammation before [7], we were keen to study the effect of anti-TNF- $\alpha$ (Infliximab) [8] pre-treatment in mice undergoing gentle liver manipulation. Whereas gentle liver mobilization resulted in acute hepatic inflammation, Infliximab pretreatment in our current settings was not able to protect mice from mobilization-induced liver inflammation. Our data show that our model provides an excellent opportunity to study the effects of liver mobilization in vivo. Further studies are needed to investigate whether anti-inflammatory mediators can be used to prevent liver inflammation following liver mobilization in order to improve post-operative outcome.

\section{Materials and Methods}

\subsection{Mice}

All animal experiments were conducted according to Home Office guidelines under the UK Animals in Scientific Procedures Act 1986. Ten- to twelve-week old C57BL6 male mice were obtained from Charles Rivers (Margate, UK). The mice were housed in the comparative biological unit at the Royal Fee and University College Medical School, University College London at least a week before start of the experiments. They were given free access to standard chow (RM1 Expanded, SDS Diets, Essex, UK) and water ad libitum, with a light/ dark cycle of 12 hours, at a temperature of $19^{\circ} \mathrm{C}$ to $23^{\circ} \mathrm{C}$ and a humidity grade of approximately $50 \%$.

\subsection{Experimental Design}

C57BL/6 mice were randomized into five groups ( $n=8$ /group) before start of the experiment. In two control groups, the liver was left alone or was subjected to laparotomy only. One group underwent surgery during which the liver was mobilized by gentle manipulation of the lobes with cotton-wool applicators for 15 minutes. Two additional groups were added that received Infliximab (10 mg/g body weight per day) by subcutaneous injection 2 days prior to surgery, one with mobilization and one without as control. Control groups received saline injection. Livers were taken, snap-frozen in liquid nitrogen and stored at $-80^{\circ} \mathrm{C}$.

\subsection{Immunohistochemistry}

Immunohistochemical stainings for inflammatory markers were performed on frozen liver sections $(7 \mu \mathrm{m})$. In 
summary, frozen liver sections were fixed in acetone and subsequently blocked for endogenous peroxidase by incubation with $0.03 \% \mathrm{H}_{2} \mathrm{O}_{2}$ for 5 minutes. After blocking with $4 \%$ fetal calf serum in $1 \times$ PBS for 30 min., sections were stained for the presence of infiltrating macrophages and neutrophils by incubating with rat-antimouse Mac-1 [M1/70] (MAB 1124; R\&D Systems; Minneapolis, USA, 1:500) and rat-anti-mouse Ly6-C [NIMP-R14] (HM1039; Hycult Biotech, Uden, the Netherlands, 1:100), respectively for $1 \mathrm{~h}$. Next, slides were incubated with polyclonal biotinylated rabbit anti rat immunoglobulins (IgG) as secondary antibody (1:200) for $1 \mathrm{~h}$ followed by peroxidase detection using the Avidin Biotin Complex (ABC) kit (PK-6100; Vector Laboratories, Burlingame, USA) for $30 \mathrm{~min}$. The 3-Amino-9-ethylcarbazole (AEC) peroxidase substrate kit (A85SK4200.S1; Bio-connect, Huissen, The Netherlands) was used as color substrate to give the red colour in presence of peroxidase. Hematoxylin (4085.9002, Klinipath, Duiven, The Netherlands) was used for nuclear counterstaining. Sections were mounted with Faramount aqueous mounting medium (S302580; DAKO, Glostrup, Denmark). Washing between all steps was performed using $1 \times$ PBS $(3 \times 3 \mathrm{~min})$. Pictures were taken with a Nikon digital camera DMX1200 and ACT-1 v2.63 software (Nikon Corporation, Tokyo, Japan). The number of positive-stained cells was counted in six pictures (200×) per liver per mouse to determine the level of infiltrating cells $/ \mathrm{mm}^{2}$ as described before [9].

\subsection{Gene Expression Analysis}

Total RNA was isolated from 25 mg of mouse liver tissue by using Tri-reagent (Sigma-Aldrich, St. Louis, USA) and was reversely transcribed into cDNA using the iScript cDNA synthesis kit (Bio-Rad, Hercules, USA), according to manufacturer's instructions. Real-time PCR was performed on a Bio-Rad MyIQ with the IQ5 v2 software using IQ SYBR Green Supermix (Bio-rad, Hercules, USA) and 10 ng of cDNA. Primers for target genes were developed using Primer Express version 2.0 [Applied Biosystems, Foster City, USA). To standardize for cDNA concentration in the samples, cyclophylin A (peptidylprolyl isomerase A, PPIA) was used as housekeeping gene. For calculations of the initial amount of mRNA present in the sample, the relative standard curve method was used.

\subsection{Surgical Procedure}

To determine the influence of gentle organ manipulation, we used a standardized protocol adapted from Schemmer et al. [3]. After 2 days of pre-treatment, all mice underwent standardized surgery. The abdomen was opened by midline incision. Dissection of livers was performed in a standardized fashion during the first minute of the procedure, during which the organ was disconnected from ligaments. Subsequently the major part of the liver was manipulated gently during 15 minutes. To maintain standard conditions, gentle manipulation was carried out with cotton-wool applicators by touching the liver lobes in situ for a specific time interval uniformly and continuously. Care was taken to use the same manipulation routine and to apply the same amount of pressure in each experiment. In the control group, no manipulation was performed after opening of the abdomen. During surgery, surgeons were blinded to the allocation of the groups.

\subsection{Statistical Analysis}

Statistical analysis was performed using GraphPad Prism 5, (GraphPad Software, La Jolla, California, USA). Differences in hepatic inflammation were calculated using the unpaired t-test or one-way ANOVA with Bonferroni correction for multiple comparisons. A p-value $<0.05$ was considered statistically significant.

\section{Results}

\subsection{Gentle in Vivo Liver Mobilization Leads to Increased Hepatic Inflammation in Mice}

In order to develop a mouse model for mobilization-induced liver damage, mice underwent a procedure mimicking hepatic surgery. To investigate the inflammatory state of the liver upon this procedure, immunohistochemical stainings for inflammatory markers were performed. Compared to the control group in which the abdomen was not opened prior to collection of liver, the number of infiltrating macrophages (Mac1), neutrophils (Nimp) and T-cells (KT3) were significantly increased in mice undergoing liver mobilization (Figures 1(a)-(c)). Representative pictures for Mac1, Nimp and KT3 staining in the control versus the mobilization group are shown in Figures 2(a)-(f). Furthermore, mice with liver mobilization had increased numbers of inflammatory 


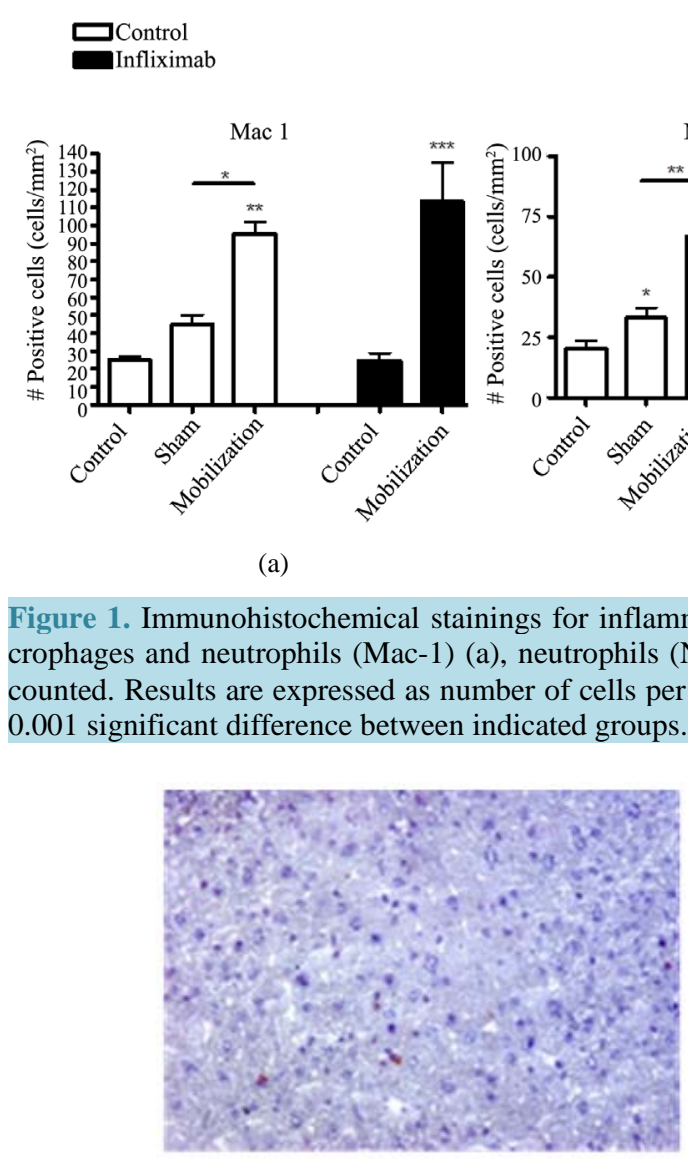

(a)

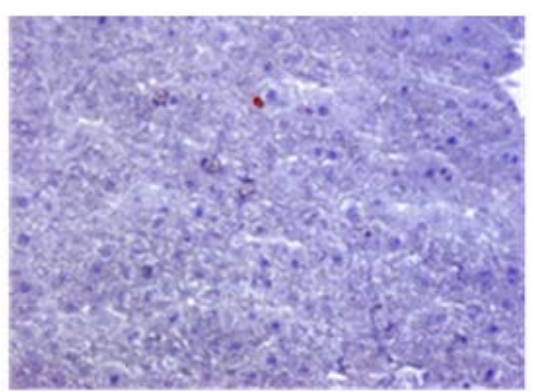

(c)

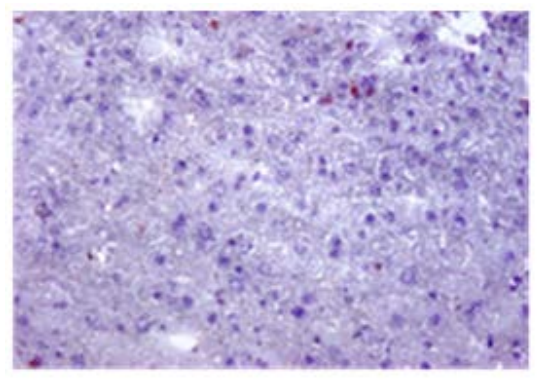

(e)

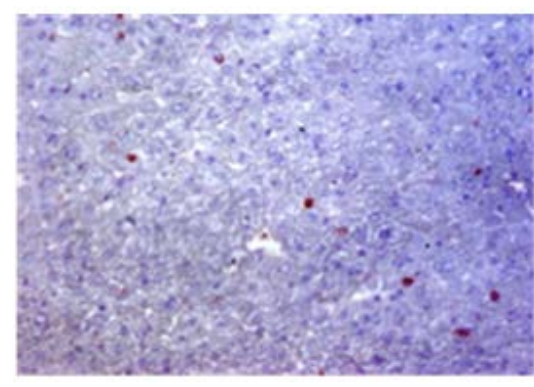

(b)

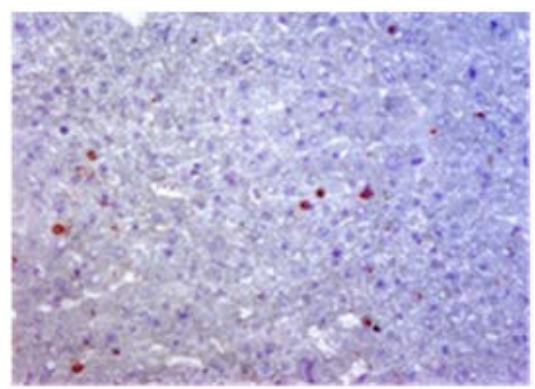

(d)

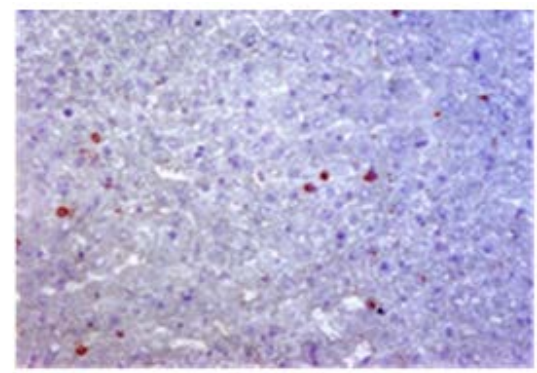

(f)

Figure 2. Immunohistochemical stainings for inflammation. Representative pictures of Mac1 (a) (b), NIMP (c) (d) and KT3 (e) (f) staining in the control group and in the group of mice undergoing liver mobilization, respectively (200× magnification). 
cells compared to mice that underwent laparotomy only as a sham treatment. Additionally, sham treated mice had only increased numbers of neutrophils compared to the control group.

To further determine whether liver mobilization affects acute inflammation, expression of genes related to inflammation was measured. In line with our immunohistochemical findings, increased inflammatory gene expression was observed in the livers of mice undergoing liver mobilization. Compared to the control group, gene expression of monocyt chemo-attractant protein $1(M c p-1)$ and interleukin-1 beta $(I L-1 \beta)$ was increased after liver mobilization (Figure 3(a) and Figure 3(b)). Furthermore, expression of $M c p-1, I L-1 \beta$ and tumor necrosis factor alpha (Tnf- $\alpha$ ) was significantly increased compared to the sham group (Figures 3(a)-(c)). Taken together, these data indicate that gentle in vivo manipulation of the liver in mice leads to an increased inflammatory response in the liver and therefore can be used as a model to investigate the underlying mechanism.

\subsection{Anti-Inflammatory Pre-Treatment Does Not Protect against Mobilization-Induced Liver inflammation.}

To investigate the potential beneficial effect of anti-inflammatory pre-treatment on mobilization-induced liver inflammation, an additional group received Infliximab two days before the surgical procedure. Infliximab pretreatment did not affect the amount of infiltrating macrophages, neutrophils and T-cells compared to saline injected mice. Whereas liver mobilization led to increased inflammation compared to controls, there was no difference observed between mice undergoing liver mobilization with saline and with Infliximab pre-treatment (Figures 1(a)-(c)).

Further, gene expression analysis was performed to study the effect of anti-inflammatory pre-treatment on inflammatory genes. Confirming our histology findings, Infliximab pre-treatment did not alter gene expression of $M c p-1, I L-1 \beta$ and $T N F-\alpha$ compared to saline injected mice undergoing mobilization (Figures 3(a)-(c)). Next to that, there was no difference observed between saline and Infliximab treatment in the control group. Taken together, these data indicate that Infliximab pre-treatment does not affect the hepatic inflammatory response during gentle manipulation of the liver in mice.

\section{Discussion}

During hepatic surgery liver mobilization results in increased liver damage associated with inflammation, causing post-operative risks (1]. So far, the potential protective effect of anti-inflammatory pre-treatment in an in vivo model to study mobilization-induced liver injury has not been investigated. To develop an appropriate in vivo mouse model, we investigated whether gentle liver manipulation in mice does trigger an inflammatory response. Additionally, the potential protective effect of anti-TNF- $\alpha$ pre-treatment using Infliximab was investigated. Our results indicate that liver mobilization results in acute liver inflammation in mice and therefore can be used as a model to investigate mobilization-induced liver inflammation to improve post-operative outcome in patients undergoing surgery. In our settings, anti-TNF- $\alpha$ pre-treatment did not prevent the development of liver inflame-

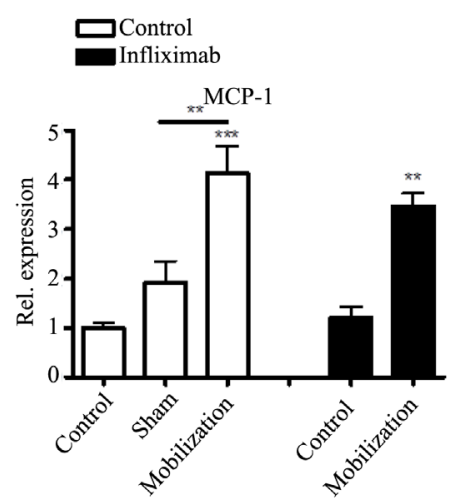

(a)

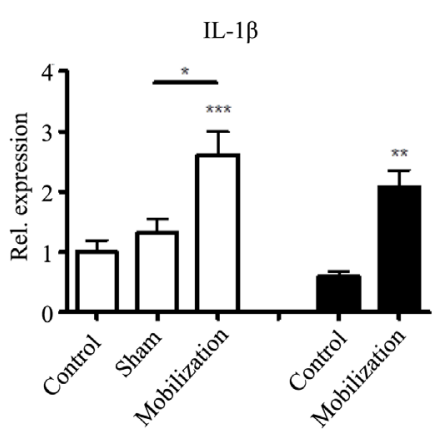

(b)

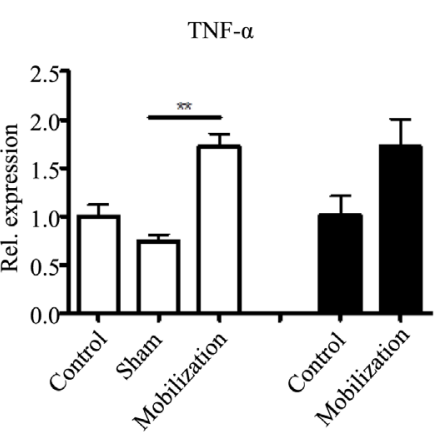

(c)

Figure 3. Hepatic inflammatory gene expression. Hepatic gene expression analysis of monocyte chemo-attractant protein 1 $(M c p-1)$, interleukin 1 beta $(I L-1 \beta)$ and tumor necrosis factor alpha $(T N F-\alpha)$. Gene expression data were set relative to control mice injected with saline. ${ }^{*}$ indicates $p<0.05$ and ${ }^{* *} p<0.01$. 
mation.

Previously, the underlying mechanism of liver damage induced by liver mobilization was investigated in a rat model for liver transplantation [1] [7]. Whereas in this study the focus was on liver damage and the role of Kupffer cells, general information regarding the inflammatory state of the liver was not described [1] [7]. Our data indicate for the first time that already $2 \mathrm{~h}$ after liver mobilization in mice increased numbers of macrophages, neutrophils and T-cells are redirected to the liver. These data are in line with our recent findings in humans, showing that inflammation is a key process after liver mobilization in patients undergoing surgery [4] [5]. Furthermore, this fast response, indicative of an increased inflammatory response, suggests that certain chemokines are released upon liver mobilization leading to increased recruitment of inflammatory cells. Further, anti-inflammatory drugs may have the potential to prevent mobilization-induced liver injury as a result of inflammation. Once activated, Kupffer cells release numerous inflammatory mediators, including tumor necrosis factor alpha (TNF- $\alpha$ ) [10]. As we were interested to study the potential beneficial effect of anti-inflammatory pretreatment on mobilization-induced liver injury, anti-TNF- $\alpha$ treatment (Infliximab) was chosen to prevent liver inflammation. However, our data did not show any significant differences between treated mice and controls. One possible explanation for this outcome may be the dosage and time-point of treatment. While it was previously shown that high dosage or long-term treatment of Infliximab may lead to different forms of liver injury in human [11] [12], our current settings consist of a lower dose (10 mg/g body weight) and short time-frame before operation. Further, it is possible that there is only a minor TNF- $\alpha$ involvement during mobilization-induced liver inflammation. Rather, the liver mobilization induced inflammation could be attributed to another important pro-inflammatory cytokine that plays an important role during liver inflammation, namely $I L-1 \beta$. Although also other pathways are involved, the cleavage and release of $I L-1 \beta$ is partly mediated by the inflammasome, a protein complex shown to be activated during liver inflammation by cholesterol crystals [13] [14]. Therefore, inhibiting inflammasome activation or targeting $I L-1 \beta$ release may be useful to reduce the inflammatory response following liver mobilization, thereby improving post-operative outcome.

So far, data regarding the effect of anti-inflammatory pre-treatment on mobilization-induced liver inflammation is lacking. Therefore, further studies are needed to evaluate our findings and other anti-inflammatory mediators in multiple dosages should be investigated to test their potential usage to decrease liver inflammation during the period of mobilization. In addition, further studies are needed to investigate the potential involvement of certain chemokines in liver injury as consequence of liver-mobilization, thereby potentially identifying new targets for prevention of mobilization-induced liver inflammation. The mouse model we established gives us the opportunity to further investigate surgery related liver damage and post-resection liver failure. This will be beneficial to improve the safety of liver surgery and to diminish graft damage during organ retrieval.

\section{Acknowledgements}

Infliximab was kindly provided by staff of the Infusion Clinic of the University College London Hospital, London, United Kingdom.

\section{Funding}

Liliane Mpabanzi was supported by a grant of the Netherlands Organization for Scientific Research (NOW Mosaic, grant $\mathrm{nr}$ 017-006-106).

\section{References}

[1] Schemmer, P., Bradford, B.U., Bunzendahl, H., Lemasters, J.J. and Thurman, R.G. (2000) Gentle in Situ Liver Manipulation during Organ Harvest Increases Oxygen Consumption in Liver. Transplantation Proceedings, 32, 112. http://dx.doi.org/10.1016/S0041-1345(99)00902-1

[2] Schemmer, P., Connor, H.D., Arteel, G.E., et al. (1999) Reperfusion Injury in livers Due to Gentle in Situ Organ Manipulation during Harvest Involves Hypoxia and Free Radicals. The Journal of Pharmacology and Experimental Therapeutics, 290, 235-240.

[3] Schemmer, P., Schoonhoven, R., Swenberg, J.A., Bunzendahl, H. and Thurman, R.G. (1998) Gentle in Situ Liver Manipulation during Organ Harvest Decreases Survival after Rat Liver Transplantation: Role of Kupffer Cells. Transplantation, 65, 1015-1020. http://dx.doi.org/10.1097/00007890-199804270-00001

[4] Van Den Broek, M.A., Shiri-Sverdlov, R., Schreurs, J.J., et al. (2013) Liver Manipulation during Liver Surgery in 
Humans is Associated with Hepatocellular Damage and Hepatic Inflammation. Liver International: Official Journal of the International Association for the Study of the Liver, 33, 633-641. http://dx.doi.org/10.1111/liv.12051

[5] Van de Poll, M.C., Derikx, J.P., Buurman, W.A., et al. (2007) Liver Manipulation Causes Hepatocyte Injury and Precedes Systemic Inflammation in Patients Undergoing Liver Resection. World Journal of Surgery, 31, 2033-2038. http://dx.doi.org/10.1007/s00268-007-9182-4

[6] Liaskou, E., Wilson, D.V. and Oo, Y.H. (2012) Innate Immune Cells in Liver Inflammation. Mediators of inflammation, 2012, Article ID: 949157. http://dx.doi.org/10.1155/2012/949157

[7] Schemmer, P., Enomoto, N., Bradford, B.U., et al. (2001) Activated Kupffer Cells Cause a Hypermetabolic State after Gentle in Situ Manipulation of Liver in Rats. American Journal of Physiology Gastrointestinal and Liver Physiology, 280, G1076-G1082.

[8] Ebert, E.C. (2009) Infliximab and the TNF-Alpha System. American Journal of Physiology Gastrointestinal and Liver Physiology, 296, G612-G620. http://dx.doi.org/10.1152/ajpgi.90576.2008

[9] Hendrikx, T., Jeurissen, M.L., Bieghs, V., et al. (2015) Hematopoietic Overexpression of Cyp27a1 Reduces Hepatic Inflammation Independently of 27-Hydroxycholesterol Levels in Ldlr(-/-) Mice. Journal of Hepatology, 62, 430-436. http://dx.doi.org/10.1016/j.jhep.2014.09.027

[10] (2012) An Inflammatory Assemblage. Nature Immunology, 13, 320. http://dx.doi.org/10.1038/ni.2268

[11] Parekh, R. and Kaur, N. (2014) Liver Injury Secondary to Anti-TNF-Alpha Therapy in Inflammatory Bowel Disease: A Case Series and Review of the Literature. Case Reports in Gastrointestinal Medicine, 2014, Article ID: 956463. http://dx.doi.org/10.1155/2014/956463

[12] Ebert, E.C., Das, K.M., Mehta, V. and Rezac, C. (2008) Non-Response to Infliximab May Be Due to Innate Neutralizing Anti-Tumour Necrosis Factor-Alpha Antibodies. Clinical and Experimental Immunology, 154, 325-31. http://dx.doi.org/10.1111/j.1365-2249.2008.03773.x

[13] Rajamaki, K., Lappalainen, J., Oorni, K., et al. (2010) Cholesterol Crystals Activate the NLRP3 Inflammasome in Human Macrophages: A Novel Link between Cholesterol Metabolism and Inflammation. PloS One, 5, Article ID: e11765. http://dx.doi.org/10.1371/journal.pone.0011765

[14] Samstad, E.O., Niyonzima, N., Nymo, S., et al. (2014) Cholesterol Crystals Induce Complement-Dependent Inflammasome Activation and Cytokine Release. Journal of Immunology, 192, 2837-2845.

http://dx.doi.org/10.4049/jimmunol.1302484 